\title{
Veränderung von Persönlichkeitsmerkmalen im Verlauf einer stationären Therapie
}

\author{
M. M. Fichter ${ }^{a, b} \quad$ N. Quadflieg ${ }^{a}$ \\ aPsychiatrische Universitätsklinik München der Ludwigs-Maximilians-Universität, Forschungsbereich Epidemiologie und Evaluation, \\ bPsychosomatische Klinik Roseneck, Prien am Chiemsee
}

\author{
Schlüsselwörter \\ Freiburger Persönlichkeitsinventar (FPI-R) · Evaluation · \\ Zeitliche Stabilität
}

\section{Zusammenfassung}

Hintergrund: Das Freiburger Persönlichkeitsinventar (FPI-R) ist ein gut etabliertes und bewährtes Verfahren zur Erfassung von Persönlichkeitseigenschaften. Im klinischen Alltag lässt sich immer wieder feststellen, dass sich trotz der postulierten Stabilität von Persönlichkeitseigenschaften im Laufe einer intensiven Therapie eindrucksvolle Veränderungen auf den Skalen des FPI-R abzeichnen.

Methode: An einer großen Stichprobe von Patienten, die stationär verhaltenstherapeutisch wegen Bulimia nervosa, Tinnitus oder einer Angststörung behandelt wurden, wurde untersucht, welche Fragen des FPI-R vor und nach der Therapie unterschiedlich bzw. gleich beantwortet wurden.

Ergebnisse: Es konnte belegt werden, dass Fragen, deren Inhalt Aspekte erfasste, die im Fokus der Therapie standen, häufiger wechselnde Antworten und damit eine größere zeitliche Instabilität aufwiesen. Die Verwendung von Konjunktiven und unbestimmten Häufigkeitswörtern in der Fragenformulierung ließen offensichtlich ein differenzierteres Abwägen von Alternativen nach der Therapie zu. Effekte einer Regression zur Mitte konnten mittels empirischer Daten ausgeschlossen werden.

Schlussfolgerung: Als Fazit lässt sich schließen, dass sich Veränderungen im Antwortverhalten vor und nach einer intensiven Therapie als spezifische Therapieeffekte erklären lassen.

\section{Key Words}

Freiburger Personality Inventory (FPI-R) - Evaluation ·

Stability over time

\section{Summary}

Change of Personality Traits in the Course of Inpatient Therapy

Background: The Freiburger Personality Inventory (FPI$\mathrm{R}$ ) is a well established and proven instrument for the assessment of personality traits. Although personality is conceived as a stable trait, clinical experience indicates that impressive changes are found on personality scales during intensive treatment.

Method: A large sample of inpatients which were treated with cognitive-behavioral therapy for bulimia nervosa, tinnitus or anxiety disorder was evaluated concerning the question which items of the FPI-R were answered differently or identically before and after intensive therapy. Results: It could be found that items which cover aspects that are central to the therapy more often show changing answers. The use of conditional form and indefinite frequency adjuncts in the formulation of items evidently allowed a more differentiated weighting of pros and cons at the end of therapy. Effects of regression to the mean could be excluded as an explanation by empirical data.

Conclusion: It can be concluded that changes in answering items before and after intensive therapy can be explained as specific effects of therapy.

\begin{tabular}{ll}
\hline KARGER & ○ 2000 S. K arger G mbH , F reiburg \\
Fax +4976145207 14 & A ccessible online at: \\
$\begin{array}{l}\text { E-mail Information@K arger.de } \\
\text { www.karger.com }\end{array}$ & www.karger.com/journals/ver
\end{tabular}




\section{Einleitung}

Nicht nur in der landläufigen Auffassung, sondern in der R egel auch in der psychologischen Forschung wird Persönlichkeit als etwas Stabiles und schwer Veränderbares angesehen (vergleiche die zusammenfassende D efinition von Herrmann [1984], der Persönlichkeit als «ein bei jedem M enschen einzigartiges, relativ stabiles und den Zeitablauf überdauerndes Verhaltenskorrelat» definiert). In den frühen Tagen der psychometrisch orientierten Persönlichkeitsforschung diskutierten Cattell und Eysenck kontrovers über die Methodik zur Erfassung von Persönlichkeit; E inigkeit bestand in der Zielsetzung: Die Erfassung stabiler Verhaltensdeterminanten, sogenannter Traits.

A llerdings gibt es $\mathrm{H}$ inweise, dass sich die Ausprägung bestimmter Persönlichkeitseigenschaften im Laufe des Lebens verändert. Conley [1984] kommt in einer Ü bersichtsarbeit zu dem Schluss, dass die Stabilitätskoeffizienten von Extraversion und $\mathrm{N}$ eurotizismus nach $10 \mathrm{~J}$ ahren bei 0,60 , nach $20 \mathrm{~J}$ ahren bei 0,40 und nach 30 Jahren bei 0,30 anzusiedeln sind, wobei hier auf eine Korrektur von korrelationsmindernden M essfehlern verzichtet wurde. Auch wenn diese Zahlen anfechtbar sind, weisen sie doch auf eine Veränderbarkeit von Persönlichkeit im Verlaufe des L ebens hin.
Klinische E rfahrungen lassen annehmen, dass Persönlichkeitseigenschaften, wie sie von Persönlichkeitstests erfasst werden, nicht nur über den gesamten L ebensverlauf Veränderungen unterliegen, sondern durch intensive $\mathrm{E}$ rlebnisse beeinflussbar sein dürften. Die vorliegende U ntersuchung befasst sich mit der Frage, welche A spekte der Persönlichkeit durch intensive neue $E$ rfahrungen, wie sie eine länger dauernde stationäre Psychotherapie darstellt, veränderbar sind und welche stabiler bleiben. U m diese Frage näher zu untersuchen, wurden Patienten vor und nach einer intensiven stationären Psychotherapie untersucht. $D$ abei wurde die Stabilität zum einen auf der Ebene der Persönlichkeitsdimensionen eines in D eutschland weit verbreiteten Persönlichkeitsinventars, des Freiburger Persönlichkeitsinventars in seiner revidierten Form (FPI-R) erfasst. Darüber hinaus untersuchten wir die Inhalte stabiler und instabiler Items dieses Instruments als kleinste Facette der erfassbaren Persönlichkeit.

\section{Methode}

Stichprobe und Procedere

A lle 367 Probanden waren Patienten, die stationär verhaltenstherapeutisch in der Medizinisch-Psychosomatischen Klinik Roseneck (Prien,

Tab. 1. G eschlecht, A Iter, Familienstand und Wohnsituation der Probanden

\begin{tabular}{|c|c|c|c|c|c|c|c|}
\hline & \multicolumn{2}{|c|}{$\begin{array}{l}\text { B ulimia nervosa } \\
(n=119)\end{array}$} & \multicolumn{2}{|c|}{$\begin{array}{l}\text { Tinnitus } \\
(n=124)\end{array}$} & \multicolumn{2}{|c|}{$\begin{array}{l}\text { A ngststörung } \\
(n=124)\end{array}$} & \multirow[t]{2}{*}{ Signifikanz } \\
\hline & $\mathrm{n}$ & $\%$ & $\mathrm{n}$ & $\%$ & $\mathrm{n}$ & $\%$ & \\
\hline \multicolumn{8}{|l|}{ G eschlecht } \\
\hline M ännlich & \multirow{2}{*}{\multicolumn{2}{|c|}{$\begin{array}{r}6 \\
113\end{array}$}} & \multicolumn{2}{|l|}{87} & \multicolumn{2}{|l|}{53} & \multirow{2}{*}{$\begin{array}{l}C h i^{2}=108,2 ; \\
d f=2 ; p<0,01\end{array}$} \\
\hline Weiblich & & & 37 & & 71 & & \\
\hline A Iter in Jahren, M W (SD ) & \multicolumn{2}{|c|}{$26,8(7,3)$} & \multicolumn{2}{|c|}{$49,8(9,3)$} & \multicolumn{2}{|c|}{$42,8(9,3)$} & $F(2 ; 364)=223,2 ; p<0,01$ \\
\hline \multicolumn{8}{|l|}{ Familienstand } \\
\hline Ledig & 86 & 72,3 & 11 & 8,9 & 15 & 12,1 & \multirow{4}{*}{$\begin{array}{l}C h i^{2}=154,3 \\
d f=8 ; p<0,01\end{array}$} \\
\hline Verheiratet & 21 & 17,6 & 95 & 76,6 & 92 & 74,2 & \\
\hline G etrennt, geschieden, verwitwet & 12 & 10,1 & 17 & 13,7 & 17 & 13,7 & \\
\hline U nbekannt & 0 & 0 & 1 & 0,8 & 0 & 0 & \\
\hline \multicolumn{8}{|l|}{ Wohnsituation } \\
\hline B ei E Itern/Verwandten & 45 & 37,8 & 3 & 2,4 & 4 & 3,2 & \multirow{5}{*}{$\begin{array}{l}C h i^{2}=159,3 \\
d f=8 ; p<0,01\end{array}$} \\
\hline Wohngemeinschaft o.ä. & 11 & 9,2 & 1 & 0,8 & 0 & 0 & \\
\hline Partner/E hemann/Familie & 28 & 23,5 & 105 & 84,7 & 110 & 88,7 & \\
\hline A llein & 35 & 29,4 & 14 & 11,3 & 10 & 8,1 & \\
\hline U nbekannt & 0 & 0 & 1 & 0,8 & 0 & 0 & \\
\hline \multicolumn{8}{|l|}{ Schulabschluss } \\
\hline H auptschule & 23 & 19,3 & 40 & 32,3 & 52 & 41,9 & \multirow{6}{*}{$\begin{array}{l}C h i^{2}=81,3 \\
d f=12 ; p<0,01\end{array}$} \\
\hline M ittlere R eife/Fachschule & 47 & 39,5 & 58 & 46,8 & 44 & 35,5 & \\
\hline A bitur & 38 & 31,9 & 2 & 1,6 & 7 & 5,6 & \\
\hline Studium ohne A bschluss & 6 & 5,0 & 2 & 1,6 & 3 & 2,4 & \\
\hline Studium mit A bschluss & 4 & 3,4 & 20 & 16,1 & 16 & 12,9 & \\
\hline A ndere & 1 & 0,8 & 2 & 1,6 & 2 & 1,6 & \\
\hline
\end{tabular}


D eutschland) behandelt wurden. D ie 119 Patienten der E ssstörungsgruppe waren zwischen 1986 und 1989 konsekutiv in der K linik aufgenommen worden. Sie hatten, gemäß dem D iagnostischen und Statistischen M anual Psychischer Störungen (D SM -III-R) [A PA, 1987] Bulimia nervosa. Die Tinnitusgruppe bestand aus 124 Personen, die zwischen A pril 1992 und A pril 1994 aufgenommen wurden. A lle Patienten dieser Gruppe hatten die ICD-Diagnose eines chronisch komplexen Tinnitus aurium. 77 der Tinnitus-Patienten hatten daneben eine oder mehrere psychiatrische D iagnosen; die häufigste Komorbiditätsdiagnose war aus dem B ereich der affektiven Störungen: M ajor D epression (30 Fälle), depressive Störung NNB (27 Fälle) und D ysthymie (22 Fälle). D ie 124 Patienten der A ngststörungsgruppe begannen zwischen Januar 1992 und A pril 1994 eine stationäre B ehandlung in R oseneck. 70 dieser Patienten hatten eine Panikstörung mit A goraphobie, 16 eine Panikstörung ohne A goraphobie, 16 eine soziale Phobie, 8 eine $Z$ wangsstörung und 6 eine $A$ goraphobie ohne Panikstörung und die restlichen eine nicht näher bezeichnete $A$ ngststörung.

Tabelle 1 stellt soziodemographische Variablen für die drei Patientengruppen dar. E rwartungsgemäß war die Essstörungsgruppe jünger, bestand überwiegend aus Frauen, war relativ häufig unverheiratet, wohnte relativ häufig bei den Eltern und hatte die längste B ehandlungsdauer. D ie beiden anderen Gruppen unterschieden sich dagegen nur unwesentlich, wobei sich alle drei G ruppen bezüglich des A Iters signifikant voneinander unterschieden. In diesem Zusammenhang ist darauf hinzuweisen, dass A Iter und G eschlecht bei der Transformation der Rohwerte in Stanine berücksichtigt werden.

Instrumente

A lle Patienten füllten in der ersten und letzten Woche ihres stationären A ufenthaltes das Freiburger Persönlichkeitsinventar in seiner revidierten Fassung (FPI-R ) [Fahrenberg et al., 1984; 1994] aus. D as FPI-R beinhaltet die Skalen FPI-R 1 - Lebenszufriedenheit, FPI-R 2 - Soziale O rientierung, FPI-R 3 - Leistungsorientierung, FPI-R 4 - Gehemmtheit, FPI-R 5 E rregbarkeit, FPI-R 6 - A ggressivität, FPI-R 7 - B eanspruchung, FPI-R 8 - Körperliche B eschwerden, FPI-R 9 - G esundheitssorgen, FPI-R $100 \mathrm{f}$ fenheit, FPI-R E - Extraversion und FPI-R N - E motionalität. Die Skalenwerte werden als Stanine ausgedrückt, mit dem M ittelwert 5. A Is N ormalbereich wird der Bereich zwischen 4 und 6 angesehen. Der Fragebogen besteht insgesamt aus 138 A ussagen, bei denen der B efragte jeweils entscheiden muss, ob dieses für inn zutrifft oder nicht (dichotome A ntwortskala).

Von allen Patienten lagen Informationen aus der B asisdokumentation der K linik R oseneck (soziodemographische A ngaben, Therapie, Therapieverlauf) vor. Weitere $D$ aten, die vor allem zur diagnostischen Einordnung der Probanden herangezogen wurden, wurden aus dieser B asisdokumentation und, bei der E ssstörungsgruppe, aus einem ausführlichen klinikspezifischen Fragebogen gewonnen.

D esign

$D$ ie A rbeit legt den Schwerpunkt exemplarisch auf die Darstellung von E rgebnissen zum FPI-R bei den Patienten, die wegen einer Bulimia nervosa behandelt wurden, da bei dieser $\mathrm{G}$ ruppe die A ussage der A rbeit am besten darstellbar ist. D ie Patienten mit Tinnitus oder einer A ngststörung dienen Vergleichszwecken. A us diesem $G$ rund werden die $E$ rgebnisse der beiden anderen $\mathrm{G}$ ruppen nur in B ezug zu Bulimia nervosa berichtet und diskutiert, um einen ersten Interpretationsrahmen zu bieten. E ine eingehende B earbeitung der Thematik bei den beiden anderen Störungsgruppen würde voraussetzen, dass die in den Tabellen 3 und 4 dargestellten A nalysen für stabile und instabile I tems dieser $\mathrm{G}$ ruppen wiederholt würden, was den $\mathrm{R}$ ahmen dieser $\mathrm{A}$ rbeit sprengen würde.
G egenstand der A nalyse ist die Veränderung von FPI-R -Persönlichkeitseigenschaften vom Zeitpunkt der A ufnahme bis zur Entlassung aus der Klinik.

D ie Signifikanzwerte der t-Tests der berichteten Veränderungen von A ufnahme nach $E$ ntlassung wurden durch Teilung des kritischen $p$-Wertes $(0,05)$ durch 12 (Zahl der Skalen des FPI-R) alpha-korrigiert.

\section{Ergebnisse}

\section{Veränderung der Skalenwerte während der Therapie}

Tabelle 2 gibt einen Ü berblick über die Skalenwerte bei A ufnahme und E ntlassung aus stationärer Therapie.

$B$ ei Patientinnen und Patienten mit Bulimia nervosa sind Veränderungen bei A ggressivität, B eanspruchung, G esundheitssorgen und $\mathrm{O}$ ffenheit nicht signifikant, die Veränderungen in den restlichen Skalen (im Sinne einer Normalisierung) sind hoch signifikant. D amit stellen sich die Bulimia-nervosa-Patienten bei Entlassung zwar als signifikant zufriedener als bei A ufnahme dar, sie bleiben aber doch deutlich mit ihrem Leben unzufrieden. Im Verlauf der B ehandlung verändern sich die Werte der Skalen Soziale Orientierung, Gehemmtheit, E rregbarkeit, Körperliche B eschwerden, E motionalität, Leistungsorientierung und Extraversion. B emerkenswert ist, dass die Skalen E rregbarkeit, Körperliche B eschwerden und Emotionalität trotz deutlicher Verringerung im Laufe der Therapie bei Entlassung noch über den Normbereich hinaus erhöht sind.

In der G ruppe der Patienten mit Tinnitus zeigten sich signifikante Veränderungen über die Therapie bei Leistungsorientierung und Extraversion, welche aber zu beiden M esszeitpunkten innerhalb des $\mathrm{N}$ ormbereichs waren. Bemerkenswert in dieser $\mathrm{G}$ ruppe war, dass sie für $\mathrm{E}$ rregbarkeit und B eanspruchung bei $A$ ufnahme und $E$ ntlassung erhöhte Werte zeigte.

D ie G ruppe der Patienten mit Angststörungen zeigte signifikante Veränderungen im Verlauf der Therapie bei den Skalen Lebenszufriedenheit, L eistungsorientierung, G ehemmtheit, Extraversion und E motionalität. A uffällig sind die geringe L ebenszufriedenheit, hohe emotionale Labilität und stark ausgeprägte $\mathrm{G}$ ehemmtheit, deren Werte bei $\mathrm{A}$ ufnahme und $\mathrm{E}$ ntlassung außerhalb der N orm lagen.

A Is globales M aß der zeitlichen Stabilität der FPI-R-Skalen über die stationäre $B$ ehandlung hinweg wurden Korrelationskoeffizienten nach Pearson berechnet. B ei Patienten mit Bulimia nervosa fand sich lediglich in der Skala G esundheitssorgen eine Korrelation über 0,70, während dies bei den Patienten mit Tinnitus mit Ausnahme von A ggressivität $(0,68)$ und G esundheitssorgen $(0,67)$ bei fast allen Skalen so war, ebenso wie bei Patienten mit A ngststörungen, bei denen nur die Skalen B eanspruchung $(0,69)$ und $E$ motionalität $(0,67)$ unter 0,70 blieben. Bei den bulimischen Patienten waren 3 Skalen (Lebenszufriedenheit 0,33 , Beanspruchung 0,49 und $E$ motionalität 0,40 ) von geringer Stabilität. Vergleichbare E rgebnisse zeigt die B erechnung der $\mathrm{E}$ ffektstärken (Formel s. Tab. 2) für 
Tab. 2. Veränderung des FPI-R im Verlauf einer stationären Psychotherapie

\begin{tabular}{|c|c|c|c|c|c|c|c|}
\hline \multirow[t]{2}{*}{ FPI-Skala } & \multirow[t]{2}{*}{$r$} & \multicolumn{2}{|c|}{ A ufnahme } & \multicolumn{2}{|c|}{ Entlassung } & \multirow{2}{*}{$\begin{array}{l}\text { t-Test (A ufnahme } \\
\text { vs. E ntlassung) }\end{array}$} & \multirow[t]{2}{*}{ E ffektstärk€ } \\
\hline & & MW & SD & M W & SD & & \\
\hline \multicolumn{8}{|c|}{$\begin{array}{l}\text { Patienten mit Bulimia nervosa } \\
\qquad(\mathrm{n}=119, \mathrm{df}=118)\end{array}$} \\
\hline Lebenszufriedenheit & 0,33 & 2,4 & 1,1 & 3,2 & 1,4 & $-5,8^{*}$ & 0,73 \\
\hline Soziale O rientierung & 0,66 & 5,5 & 1,7 & 4,8 & 1,9 & $4,7^{*}$ & $-0,41$ \\
\hline L eistungsorientierung & 0,67 & 4,0 & 1,9 & 4,8 & 1,8 & $-5,7^{*}$ & 0,42 \\
\hline G ehemmtheit & 0,60 & 6,8 & 2,0 & 5,7 & 2,0 & $6,3^{*}$ & $-0,55$ \\
\hline E rregbarkeit & 0,52 & 7,0 & 1,5 & 6,3 & 1,9 & $4,4^{*}$ & $-0,47$ \\
\hline A ggressivität & 0,54 & 4,8 & 1,7 & 5,2 & 1,5 & $-2,5$ & 0,24 \\
\hline B eanspruchung & 0,49 & 6,1 & 1,3 & 5,7 & 1,7 & 2,6 & $-0,31$ \\
\hline K örperliche B eschwerden & 0,57 & 7,0 & 1,7 & 6,2 & 1,8 & $-5,6^{*}$ & $-0,47$ \\
\hline G esundheitssorgen & 0,74 & 4,1 & 1,8 & 4,4 & 1,9 & $-2,8$ & 0,17 \\
\hline O ffenheit & 0,59 & 5,7 & 1,8 & 5,5 & 1,8 & 1,2 & $-0,11$ \\
\hline Extraversion & 0,64 & 3,4 & 1,8 & 4,5 & 2,0 & $-7,4^{*}$ & 0,61 \\
\hline E motionalität & 0,40 & 7,6 & 1,5 & 6,7 & 1,9 & $5,2^{*}$ & $-0,60$ \\
\hline \multicolumn{8}{|l|}{$\begin{array}{l}\text { Patienten mit Tinnitus } \\
\qquad(\mathrm{n}=124, \mathrm{df}=123)\end{array}$} \\
\hline L ebenszufriedenheit & 0,79 & 4,2 & 2,1 & 4,4 & 2,2 & $-1,4$ & 0,10 \\
\hline Soziale O rientierung & 0,74 & 6,0 & 1,8 & 5,8 & 1,8 & 1,4 & $-0,11$ \\
\hline L eistungsorientierung & 0,74 & 4,5 & 1,6 & 4,8 & 1,8 & $-3,5^{*}$ & 0,19 \\
\hline G ehemmtheit & 0,77 & 5,7 & 2,2 & 5,4 & 2,1 & 2,6 & $-0,14$ \\
\hline E rregbarkeit & 0,72 & 6,5 & 2,0 & 6,1 & 2,1 & 2,9 & $-0,20$ \\
\hline A ggressivität & 0,68 & 4,4 & 1,9 & 4,5 & 2,9 & $-1,2$ & 0,05 \\
\hline B eanspruchung & 0,71 & 6,2 & 1,8 & 6,2 & 1,9 & $-0,1$ & 0,00 \\
\hline K örperliche B eschwerden & 0,76 & 5,5 & 1,8 & 5,4 & 1,9 & 0,8 & $-0,06$ \\
\hline G esundheitssorgen & 0,67 & 5,1 & 1,5 & 5,1 & 1,6 & $-0,2$ & 0,00 \\
\hline Offenheit & 0,72 & 4,7 & 1,9 & 4,8 & 1,9 & $-0,6$ & 0,05 \\
\hline Extraversion & 0,80 & 4,0 & 2,0 & 4,5 & 2,0 & $-4,1^{*}$ & 0,25 \\
\hline E motionalität & 0,73 & 6,1 & 2,0 & 5,9 & 2,0 & 1,8 & $-0,10$ \\
\hline \multicolumn{8}{|l|}{$\begin{array}{l}\text { Patienten mit A ngststörung } \\
\qquad(\mathrm{n}=124, \mathrm{df}=123)\end{array}$} \\
\hline L ebenszufriedenheit & 0,78 & 3,1 & 1,7 & 3,5 & 1,9 & $-3,7^{*}$ & 0,24 \\
\hline Soziale O rientierung & 0,75 & 5,6 & 1,9 & 5,5 & 2,1 & 1,2 & $-0,05$ \\
\hline L eistungsorientierung & 0,82 & 3,9 & 1,7 & 4,5 & 1,8 & $-5,7^{*}$ & 0,35 \\
\hline G ehemmtheit & 0,77 & 6,9 & 1,8 & 6,2 & 2,0 & $6,3^{*}$ & $-0,39$ \\
\hline E rregbarkeit & 0,73 & 7,1 & 1,8 & 6,8 & 2,0 & 2,5 & $-0,17$ \\
\hline A ggressivität & 0,73 & 4,7 & 1,8 & 4,7 & 2,0 & 0,1 & 0,00 \\
\hline B eanspruchung & 0,69 & 6,3 & 1,8 & 6,3 & 1,8 & $-0,6$ & 0,00 \\
\hline K örperliche B eschwerden & 0,75 & 7,0 & 1,6 & 6,7 & 1,7 & 2,7 & $-0,19$ \\
\hline G esundheitssorgen & 0,77 & 5,1 & 1,8 & 5,1 & 1,8 & $-0,4$ & 0,00 \\
\hline Offenheit & 0,71 & 5,2 & 1,8 & 5,2 & 1,9 & 0,1 & 0,00 \\
\hline Extraversion & 0,76 & 3,4 & 1,8 & 4,3 & 2,0 & $-8,2^{*}$ & 0,50 \\
\hline E motionalität & 0,67 & 7,5 & 1,4 & 7,1 & 1,8 & $3,9^{*}$ & $-0,29$ \\
\hline
\end{tabular}

$r=$ Pearsons Produkt-M oments-Korrelationskoeffizient «A ufnahme-E ntlassung», df = Freiheitsgrade, $M$ W = M ittelwert, SD =Standardabweichung. Formel für E ffektstärke: (M ittelwert E ntlassung - M ittelwert A ufnahme) / SD A ufnahme.

$\mathrm{df}=$ Freiheitsgrade, $\mathrm{M} \mathrm{W}=\mathrm{M}$ ittelwert, $\mathrm{SD}=$ Standardabweichung.

$* p<0,004$. 
jede FPI-Skala getrennt nach G ruppen. Während diese in den beiden nicht-bulimischen $G$ ruppen in der überwiegenden $M$ ehrzahl sehr gering ausfallen (A usnahme ist E motionalität bei $A$ ngststörungen mit 0,50 ), sind in der bulimischen $G$ ruppe bei 4 Skalen (L ebenszufriedenheit, $G$ ehemmtheit, Extraversion und $\mathrm{E}$ motionalität) $\mathrm{E}$ ffektstärken über 0,50 zu beobachten.

Veränderung des A ntwortverhaltens bei A ufnahme und E ntlassung auf I tem-E bene

B ei einer zweimaligen B efragung mit dem FPI-R sind 3 Varianten an A ntwortverhalten möglich: Zum einen kann zu beiden Zeitpunkten für ein Item die gleiche A ntwort (also «stimmt» bei Beginn und Ende der Therapie oder «stimmt nicht» bei $B$ eginn und $E$ nde der Therapie) gegeben werden (M öglichkeit A ), zum anderen kann bei B eginn der Therapie mit «stimmt nicht» und zu Ende der Therapie mit «stimmt» geantwortet werden (M öglichkeit B), des weiteren ist die Möglichkeit gegeben, bei Beginn der Therapie mit «stimmt» und zu Ende der Therapie mit «stimmt nicht» zu antworten (M öglichkeit $C$ ). In einem ersten A nalyseschritt wurde für jeden Patienten und für jedes Item bestimmt, ob die A ntwort bei B eginn und $E$ nde der Therapie gleich war (Möglichkeit A ) oder ob sie unterschiedlich ausfiel (M öglichkeit B oder C). A Is Index der Stabilität eines I tems wurde der Prozentsatz der Personen herangezogen, welche bei Beginn und $E$ nde der Therapie bei dem jeweiligen Item die gleiche A ntwort gaben. Folgende Resultate konnten gefunden werden: 4 Items bei Bulimia nervosa bzw. 0 bei Tinnitus und 0 bei A ngststörungen wiesen einen geringen Stabilitätsindex zwischen 58 und $60 \%$ auf. E inen Stabilitätsindex von $61-70 \%$ hatten 26 bzw. 3 bzw. 6 I tems. 80 bzw. 74 bzw. 64 I tems lagen zwischen 71 und $80 \%$, 25 bzw. 60 bzw. 68 I tems blieben zu 81-90\% stabil und im Bereich von 91 bis $100 \%$ waren 3 bzw. 1 bzw. 0 I tems und wiesen damit auf eine hohe Stabilität hin. Der chi $^{2}$-Wert für eine $K$ reuztabelle der drei $D$ iagnosegruppen und fünf $K$ lassen des Stabilitätsindex ( $\mathrm{df}=8$ ) ist mit 60,61 hoch signifikant ( $p<$ 0,01 ). Insgesamt sind die A ntworten in der Tinnitus- und in der A ngststörungsgruppe stabiler als bei Patienten mit Bulimia nervosa, wobei allerdings auffällt, dass bei den E ssstörungen im Vergleich zu den anderen $\mathrm{G}$ ruppen mehr Items sowohl in der niedrigsten als auch in der höchsten $\mathrm{K}$ ategorie zu finden sind.

Für das weitere Vorgehen wurden zwei G ruppen von FPI-R Items gebildet. A Is instabile Items wurden diejenigen definiert, die in der Bulimia-nervosa-G ruppe einen Stabilitätsindex von höchstens $70 \%$ aufwiesen. D ies waren 30 Items. Stabile I tems waren 28 Items mit einem Stabilitätsindex von mindestens 80,5 - aufgerundet $81 \%$ (vgl. Tab. 3).

Vergleicht man, zu welchen FPI-R -Skalen die Items gehören, so war ein I tem ohne Skalenzuordnung, 1 instabiles und 4 stabile I tems gehörten zur Skala L ebenszufriedenheit, 2 instabile und 3 stabile Items zu Soziale 0 rientierung, 5 instabile und 0 stabile I tems zu Leistungsorientierung, 3 instabile und 0 stabile Items zu G ehemmtheit, 2 instabile und 2 stabile Items zu E rregbarkeit, 3 instabile und 4 stabile I tems zu A ggressivität, 3 instabile und 1 stabiles I tem zu Beanspruchung, 4 instabile und 1 stabiles Item zu Körperliche B eschwerden, 0 instabile und 6 stabile Items zu G esundheitssorgen, 1 instabiles und 3 stabile I tems zu 0 ffenheit, 2 instabile und 2 stabile I tems zu Extraversion und 4 instabile und 1 stabiles I tem zu E motionalität. Der chi²-Test ( $\mathrm{df}=12$ ) ist mit einem Wert von 22,70 signifikant $(p<0,05)$. Items zur L eistungsorientierung, $G$ ehemmtheit, $B$ eanspruchung, $K$ örperliche $B$ eschwerden und E motionalität fallen als eher instabil und Items zu Lebenszufriedenheit, Sozialer O rientierung, G esundheitssorgen, 0 ffenheit und Extraversion als eher stabil auf. ${ }^{1}$

Betrachtet man die Richtung der Veränderung des A ntwortverhaltens bei den instabilen Items (Tab. 3), so stellen sich die Patienten, die wegen einer Bulimia nervosa behandelt wurden am Ende der Therapie als optimistischer, beschwerdenfreier, sowie emotional lebendiger und stabiler dar. In einer R eihe von A ntworten kommt ein $\mathrm{G}$ ewinn von sozialer Kompetenz und Durchsetzungsvermögen zum Ausdruck. Die stabilen Items (Tab. 4) hingegen verweisen bereits in der Formulierung auf situationsübergreifende Verhaltensweisen hin. Konkreter A usdruck dieses U nterschieds ist die $\mathrm{H}$ äufigkeit, mit der in den Items unbestimmte $\mathrm{H}$ äufigkeitsangaben gemacht werden wie z. B. häufig, oft, manchmal usw. So finden sich in 18 der 30 $(60,0 \%)$ instabilen I tems solche W örter, während dies bei den stabilen I tems nur bei 6 von 28 (21,4\%) vorkommt. B ei den 80 restlichen Items, die in $\mathrm{H}$ inblick auf die Stabilität in der $\mathrm{M}$ itte liegen, enthalten 29 Items (36,3\%) solche unbestimmte Häufigkeitsangaben.

\section{Diskussion}

Persönlichkeitseigenschaften werden in der klinisch-psychologischen Forschung in der Regel als stabile Merkmale einer Person aufgefasst, «... eine über die Zeit, im Extremfall über das ganze L eben beständige $A r t$ und Weise des $E$ rlebens einer Person in bestimmten A rten von L ebensumständen» [B randtstätter, 1989, S. 13]. Die E rgebnisse der Studie über Veränderungen von Persönlichkeitseigenschaften im Verlauf einer stationären Psychotherapie zeigen, dass dies für viele Items eines gebräuchlichen Persönlichkeitsinventars nicht zutrifft. $D$ ieses $\mathrm{E}$ rgebnis wird den $\mathrm{K}$ liniker wenig verwundern, wenn er für Persönlichkeitsskalen zumindest teilweise das Konstrukt der Stabilität von Persönlichkeitseigenschaften (Traits im $G$ egensatz zu States) infrage stellt.

\footnotetext{
${ }^{1}$ Drei instabile Items weisen eine Zuordnung zu zwei FPI-R-Skalen auf. Sie wurden für die soeben berichtete Verteilung der Skala zugeordnet, die in der Tabelle 2 zuerst genannt ist. Ordnet man die Items der zweitgenannten Skala (in allen drei Fällen Extraversion) zu, so ändern sich die Ergebnisse des $\mathrm{Chi}^{2}$ Tests praktisch nicht.
} 
Tab. 3. K ennwerte der FPI-R -I tems, deren A ntworten sich bei essgestörten Patienten (Bulimia nervosa) im L aufe einer stationären Therapie stark veränderten ${ }^{1}$

\begin{tabular}{|c|c|c|c|c|}
\hline \multirow[t]{2}{*}{ FPI-R -I tem } & \multirow[t]{2}{*}{$\begin{array}{l}\text { FPI-R -Skala, zu der } \\
\text { das I tem gehört }\end{array}$} & $\begin{array}{l}\text { Patienten, die vor der } \\
\text { Therapie mit «stimmt nicht» } \\
\text { und nach der Therapie mit } \\
\text { «stimmt» antworteten (\%) }\end{array}$ & $\begin{array}{l}\text { Patienten, die vor der } \\
\text { Therapie mit «stimmt» } \\
\text { und nach der Therapie } \\
\text { mit «stimmt nicht» } \\
\text { antworteten }(\%)\end{array}$ & \multirow[t]{2}{*}{$\begin{array}{l}\text { Chi } \\
(\mathrm{df}=4)\end{array}$} \\
\hline & & $\begin{array}{lll}\text { Bulimia } & \text { Tinni- } & \text { A ngst- } \\
\text { nervosa } & \text { tus } & \text { störung }\end{array}$ & $\begin{array}{lll}\text { Bulimia } & \text { Tinni- } & \text { A ngst- } \\
\text { nervosa } & \text { tus } & \text { störung }\end{array}$ & \\
\hline
\end{tabular}

138. M eistens blicke ich voller Zuversicht in die Zukunft

36. Ich denke oft, dass ich meinen Konsum einschränken müsste, um dann an benachteiligte $M$ enschen abzugeben

80. Ich bekomme häufig ein schlechter $\mathrm{G}$ ewissen, wenn ich sehe, wie schlecht es anderen $M$ enschen geht

13. M eine B ekannten halten mich für einen energischen $M$ enschen

43. Es gibt für mich noch eine M enge sinnvoller A ufgaben, die ich in der Zukunft anpacken werde

48. $M$ it anderen zu wetteifern, macht mir Spaß

54. B ei wichtigen $D$ ingen bin ich bereit, mit anderen energisch zu konkurrieren

101. Ich ziehe das $\mathrm{H}$ andeln dem Pläneschmieden vor

8. Ich würde mich beim $\mathrm{K}$ ellner oder $\mathrm{G}$ eschäftsführer eines R estaurants beschweren, wenn ein schlechter E ssen serviert wird

73. Ich bin im $G$ runde eher ein ängstlicher $M$ ensch

109. B ei $\mathrm{G}$ eselligkeiten und öffentlichen Veranstaltungen bleibe ich lieber im $\mathrm{H}$ intergrund

60. A uch wenn mich etwas sehr aus der Fassung bringt, beruhige ich mich meistens wieder rasch

105. Ich kann oft meinen Ä rger und meine Wut nicht beherrschen

L ebenszufriedenheit

$$
\begin{aligned}
& \text { Loz } \\
& \text { Lei } \\
& \text { Lei } \\
& \text { Lei } \\
& \text { Ext }
\end{aligned}
$$

L eistungsorientierung 21,

\section{G ehemmtheit}

G ehemmtheit

G ehemmtheit/

Extraversion

E rregbarkeit

E rregbarkeit

20. Sind wir in ausgelassener R unde, so überkommt mich oft eine große L ust zu groben Streichen

A ggressivität/

Extraversion

56. Wenn mich jemand anschreit, schreie ich zurück

90. Wenn ich wirklich wütend werde, bin ich in der $L$ age, jemanden eine runterzuhauen

35. Die täglichen B elastungen sind so groß, dass ich davon oft müde und erschöpft bin

103. Vor lauter A ufgaben und Z eitdruck bin ich manchmal ganz durcheinander

122. D ie A nforderungen, die an mich gestellt werden, sind oft zu hoch

47. I ch habe manchmal das $\mathrm{G}$ efühl, einen $\mathrm{K}$ loß im $\mathrm{H}$ als zu haben

A ggressivität

A ggressivität

B eanspruchung

35,3

10,2

0,2

10,1

8,9

5

28,2

8,1

4,2
24,6

$4,0 \quad 4,0$

$13,7 \quad 10,5$

$26,9 \quad 12,9 \quad 18,5$

22,7

$14,5 \quad 16,9$

$10,0 *$

B eanspruchung

B eanspruchung

Körperliche

B eschwerden

Körperliche

72. Ich bemerke häufiger ein unwillkürliches Zucken, z. B. um meine A ugen

99. $M$ eine $H$ ände sind häufig zittrig, $z$. B. beim A nzünden einer Zigarette oder $\mathrm{H}$ alten einer Tasse

133. Ich habe manchmal ein $\mathrm{G}$ efühl erstickender Enge in der Brust

95. M anchmal habe ich $\mathrm{G}$ edanken, über die ich mich schämen muss

Beschwerden

$12,7 \quad 8,1 \quad 12,1$

14,3

$6,5 \quad 10,5$

$13,9 * *$

$25,2 \quad 9,7 \quad 14,5$

$17,6 \quad 12,9 \quad 10,5$

$21,2 \quad 12,9 \quad 14,5 \quad$ n.s

Körperliche

B eschwerden

Körperliche

B eschwerden

$15,3 \quad 9,7 \quad 9,7$

7,6

$7,3 \quad 2,4$

$16,8 \quad 12,1 \quad 13,7$

$15,5^{* *}$

$15,3 \quad 9,7 \quad 9,7$

16,1

$11,3 \quad 12,9$

$10,9 \quad 8,9 \quad 8,1$

21,0

10,5

$12,7 \quad 10,5 \quad 10,5$

17,8

15,

$14,4 \quad 10,5 \quad 16,1$

16,1

$12,9 \quad 10,5$

$9,2 \quad 13,7 \quad 10,5$

28,6

$8,1 \quad 16,1$

$10,1 \quad 11,3 \quad 6,5$

21,0

$8,9 \quad 16,9$

n.s.

Offenheit

10,9

10,9

10,2

4,0

8,9

24,4

$6,5 \quad 13,0$

$22,4^{* *}$

$13,7 \quad 12,1$

26,3

$8,1 \quad 12,1$

$17,0 * *$

$11,9 \quad 7,3 \quad 8,1$

29,7

$12,1 \quad 22,6$

$14,8 * *$

Extraversion

24,4

14,5

23,4

9,2

5,6

$\begin{array}{ll}7,3 & \text { n.S. } \\ 4,0 & 14,1^{* *}\end{array}$

Fortsetzung S. 172 


\begin{tabular}{|c|c|c|c|c|c|c|c|c|}
\hline \multirow[t]{2}{*}{ FPI-R -Item } & \multirow[t]{2}{*}{$\begin{array}{l}\text { FPI-R -Skala, zu der } \\
\text { das Item gehört }\end{array}$} & \multicolumn{3}{|c|}{$\begin{array}{l}\text { Patienten, die vor der } \\
\text { Therapie mit «stimmt nicht» } \\
\text { und nach der Therapie mit } \\
\text { «stimmt» antworteten (\%) }\end{array}$} & \multicolumn{3}{|c|}{$\begin{array}{l}\text { Patienten, die vor der } \\
\text { Therapie mit «stimmt» } \\
\text { und nach der Therapie } \\
\text { mit «stimmt nicht» } \\
\text { antworteten (\%) }\end{array}$} & \multirow[t]{2}{*}{$\begin{array}{l}\mathrm{Chi}^{2} \\
(\mathrm{df}=4)\end{array}$} \\
\hline & & $\begin{array}{l}\text { Bulimia } \\
\text { nervosa }\end{array}$ & $\begin{array}{l}\text { Tinni- } \\
\text { tus }\end{array}$ & $\begin{array}{l}\text { A ngst- } \\
\text { störung }\end{array}$ & $\begin{array}{l}\text { Bulimia } \\
\text { nervosa }\end{array}$ & $\begin{array}{l}\text { Tinni- } \\
\text { tus }\end{array}$ & $\begin{array}{l}\text { A ngst- } \\
\text { störung }\end{array}$ & \\
\hline \multicolumn{9}{|l|}{ 19. Ich habe manchmal ein $\mathrm{G}$ efühl der } \\
\hline Teilnahmslosigkeit und inneren Leere & E motionalität & 7,6 & 12,1 & 5,6 & 22,7 & 16,1 & 21,0 & n.s. \\
\hline 42. M eine Familie und meine B ekannten können & & & & & & & & \\
\hline \multirow{4}{*}{$\begin{array}{l}\text { mich im G runde kaum richtig verstehen } \\
\text { 45. Ich fühle mich oft wie ein Pulverfass kurz vor } \\
\text { der Explosion } \\
\text { 110. Ich träume tagsüber oft von D ingen, die noch } \\
\text { nicht verwirklicht werden können }\end{array}$} & E motionalität & 13,6 & 12,1 & 11,3 & 27,1 & 10,5 & 8,9 & $20,4^{* *}$ \\
\hline & E motionalität & 13,4 & 10,5 & 12.1 & 27.7 & 14.5 & 14.5 & $107 *$ \\
\hline & & & & & & & & \\
\hline & E motionalität & 9,7 & 11,4 & 9,7 & 21,2 & 11,4 & 14,5 & n.s. \\
\hline
\end{tabular}

Zur Stabilität des in unseren U ntersuchungen verwendeten Persönlichkeitsinventars (FPI-R) im klinischen Kontext gibt es relativ wenig Literatur. Die Persönlichkeitsskalen wurden meist nur zur Beschreibung von klinischen Gruppen [z.B. Tölle et al., 1987; Böhle et al., 1991; D eter und Schepank, 1991] oder zur Vorhersage des Therapie-O utcomes [z.B. K inzl et al., 1989; H offmann et al., 1991] herangezogen. Langosch und Brodner [1984] konnten zeigen, dass die Erkrankungsdauer und das Setting bei der $D$ atenerhebung ( $Z$ uhause oder in der $\mathrm{K}$ linik) einen $\mathrm{E}$ influss auf die Persönlichkeit-Scores bei Herzinfarktpatienten haben. Fabra et al. [1991] stellte bei einer U ntersuchung zur Wirksamkeit psychiatrischer K risenintervention (D auer 4 Wochen) in der G esamtgruppe eine hohe Stabilität fest (einzig die Skala «O ffenheit» veränderte sich bedeutsam). Teusch und B öhme [1991] konnten mit einer älteren Version des FPI aufzeigen, dass sich bei Patienten mit A goraphobie und/oder Panik ein Jahr nach einer gesprächstherapeutischen B ehandlung die Bereiche Lebenszufriedenheit, G ehemmtheit, B elastung, E motionalität und $\mathrm{N}$ eurotizismus im Sinne einer Verbesserung verändert haben. Payk und Wachendorfer [1987] registrieren bei der M ehrzahl von 17 Patienten, die wegen Depression, A ngst oder Persönlichkeitsstörung tagesklinisch behandelt wurden, deutliche oder leichte Verbesserungen hinsichtlich der Skalen «D epressivität», «G eselligkeit», «Extraversion» und «emotionale Stabilität». A uf eine statistische A bsicherung inrer E rgebnisse verzichten sie. Künsebeck et al. [1987] verzeichnen A uswirkungen von begleitender Psychotherapie bei Patienten mit M orbus Crohn auf die Skalen «Nervosität» und «D epressivität» (verwendet wurde eine ältere Version des FPI), aller- dings auch behandlungsunabhängige Z eiteffekte auf die Skalen «A ggressivität», «D epressivität» und «G eselligkeit». Paul und Jacobi [1986] konnten im R ahmen eines ambulanten Behandlungsprogramms bei Bulimia nervosa recht hohe Stabilität feststellen (FPI-K). Lediglich die Skala «D epressivität» verzeichnete einen bedeutsamen $R$ ückgang. Fahrenberg et al. [1994] berichten Stabilitätskoeffizienten zwischen dem B eginn und dem $\mathrm{E}$ nde einer Kur bei 103 männlichen $\mathrm{H}$ erz-K reislaufPatienten in $\mathrm{H}$ öhe von 0,63 (Soziale O rientierung) bis 0,85 ( $G$ ehemmtheit). D ie Stabilitätskoeffizienten bei Patienten mit Tinnitus oder A ngststörungen in der vorliegenden U ntersuchung liegen in einem vergleichbaren $\mathrm{R}$ ahmen, die der Patienten mit Bulimia nervosa deutlich darunter. $\mathrm{G}$ leiches zeigen die berichteten $\mathrm{E}$ ffektstärken. $\mathrm{H}$ ierbei zeichnen sich besonders L ebenszufriedenheit, $G$ ehemmtheit, Extraversion und $E$ motionalität therapeutisch als besonders gut beeinflussbar aus. Vergleichbar hohe E ffektstärken fanden sich bei Tinnitus- und A ngststörungspatienten nicht.

$\mathrm{N}$ ach den $\mathrm{E}$ rgebnissen unserer U ntersuchung unterschieden sich die drei untersuchten Patientengruppen hinsichtlich der Stabilität von Persönlichkeitseigenschaften, wie sie mit dem FPI-R erfasst werden. D ie Patienten der E ssstörungsgruppe (Bulimia nervosa) zeigten deutlichere Veränderungen als die Patienten der beiden anderen $\mathrm{G}$ ruppen. B edeutsam ist hier, dass bei der Essstörungsgruppe die Mittelwertsunterschiede größer sind, verglichen mit den beiden anderen Gruppen. Diese Ergebnisse dürften zumindest teilweise durch die A Itersunterschiede zwischen den G ruppen erklärbar sein. Die E ssstörungsgruppe war im D urchschnitt deutlich jünger als die beiden anderen G ruppen. A us der Persönlichkeitspsychologie 
Tab. 4. K ennwerte der FPI-R -Items, deren A ntworten sich bei essgestörten Patienten (Bulimia nervosa) im L aufe einer stationären Therapie wenig veränderten ${ }^{1}$

\begin{tabular}{|c|c|c|c|c|c|c|c|}
\hline \multirow[t]{2}{*}{ FPI-R -I tem } & \multirow[t]{2}{*}{$\begin{array}{l}\text { FPI-R-Skala, zu der } \\
\text { das I tem gehört }\end{array}$} & \multicolumn{2}{|c|}{$\begin{array}{l}\text { Patienten, die vor der } \\
\text { Therapie mit «stimmt nicht» } \\
\text { und nach der Therapie mit } \\
\text { «stimmt» antworteten (\%) }\end{array}$} & \multicolumn{3}{|c|}{$\begin{array}{l}\text { Patienten, die vor der } \\
\text { Therapie mit «stimmt» } \\
\text { und nach der Therapie } \\
\text { mit «stimmt nicht» } \\
\text { antworteten (\%) }\end{array}$} & \multirow[t]{2}{*}{$\begin{array}{l}\mathrm{Chi}^{2} \\
(\mathrm{df}=4)\end{array}$} \\
\hline & & $\begin{array}{ll}\text { Bulimia Tinni- } \\
\text { nervosa tus }\end{array}$ & $\begin{array}{l}\text { A ngst- } \\
\text { störung }\end{array}$ & $\begin{array}{l}\text { Bulimia } \\
\text { nervosa }\end{array}$ & $\begin{array}{l}\text { Tinni- } \\
\text { tus }\end{array}$ & $\begin{array}{l}\text { A ngst- } \\
\text { störung }\end{array}$ & \\
\hline
\end{tabular}

1. Ich habe die A nleitung gelesen und bin bereit, jeden Satz offen zu beantworten

23. Ich lebe mit mir selbst in Frieden und ohne innere Konflikte

88. Ich bin immer guter $L$ aune

128. A lles in allem bin ich ausgesprochen zufrieden mit meinem bisherigen Leben

131. M eine Partnerbeziehung ( $E$ he) ist gut

41. Wenn jemand weint, möchte ich ihn am liebsten umarmen und trösten

134. Ich habe schon unbezahlt beim R oten $\mathrm{K}$ reuz, in meiner $\mathrm{G}$ emeinde oder in anderen sozialen Einrichtungen geholfen

136. Ich nehme mir viel Z eit, anderen $M$ enschen geduldig zuzuhören, wenn sie von ihren Sorgen erzählen

27. I ch neige dazu, bei A useinandersetzungen lauter zu sprechen als sonst

52. Auch wenn es eher viel zu tun gibt, lasse ich mich nicht hetzen

15. Ich kann mich erinnern, mal so zornig gewesen zu sein, dass ich das nächstbeste D ing nahm und es zerriss oder zerschlug

37. A Is K ind habe ich manchmal ganz gerne anderen die $\mathrm{A}$ rme umgedreht, an $\mathrm{H}$ aaren gezogen, ein B ein gestellt usw.

40. Es macht mir Spaß, anderen Fehler nachzuweisen

50. Wenn ich Zuflucht zu körperlicher $\mathrm{G}$ ewalt nehmen muss, um meine $R$ echte zu verteidigen, so tue ich es

28. Ich bin oft nervös, weil zu viel auf mich einströmt

16. Ich habe häufig Kopfschmerzen

38. U m gesund zu bleiben, achte ich auf ein ruhiges $L$ eben

62. Ich vermeide es, ungewaschenes 0 bst zu essen

65. Ich vermeide $Z$ ugluft, weil man sich zu leicht erkälten kann

84. Weil man sich so leicht anstecken kann, wasche ich mir zu $\mathrm{H}$ ause gleich die $\mathrm{H}$ ände

89. Ich passe auf, dass ich nicht zuviel A utoabgase und Staub einatme

127. A uch ohne ernste B eschwerden gehe ich regelmäßig zum $A$ rzt, nur zur Vorsicht

66. $M$ anchmal schiebe ich etwas auf, was ich sofort tun sollte

77. M anchmal bin ich beleidigt, wenn es nicht nach meinem Willen geht

114. Ich spreche manchmal über D inge, von denen ich nichts verstehe

\begin{tabular}{|c|c|c|c|c|c|c|c|}
\hline & 0,0 & 0,0 & 2,4 & 0,9 & 0,0 & 1,6 & n.s. \\
\hline Lebenszufriedenheit & 7,6 & 13,7 & 12,9 & 0,0 & 7,3 & 3,2 & $12,8^{*}$ \\
\hline L ebenszufriedenheit & 5,9 & 6,5 & 6,5 & 5,9 & 3,2 & 0,8 & n.s. \\
\hline L ebenszufriedenheit & 10,1 & 11,3 & 12,9 & 9,2 & 8,1 & 9,7 & n.s. \\
\hline L ebenszufriedenheit & 15,0 & 5,6 & 11,4 & 4,0 & 8,1 & 4,9 & n.s. \\
\hline Soziale 0 rientierung & 2,5 & 8,1 & 8,1 & 14,4 & 9,7 & 10,5 & n.s. \\
\hline Soziale 0 rientierung & 11,0 & 6,5 & 8,9 & 8,5 & 4,0 & 5,6 & n.s. \\
\hline Soziale 0 rientierung & 5,9 & 7,3 & 10,5 & 11,8 & 6,5 & 7,3 & n.s. \\
\hline E rregbarkeit & 10,9 & 10,5 & 8,9 & 8,4 & 9,7 & 8,1 & n.s. \\
\hline E rregbarkeit & 15,1 & 8,1 & 11,3 & 2,5 & 3,2 & 8,9 & n.s. \\
\hline A ggressivität & 9,2 & 10,5 & 8,1 & 10,1 & 10,5 & 10,5 & n.s. \\
\hline A ggressivität & 13,6 & 5,6 & 13,7 & 5,1 & 4,0 & 4,8 & n.s. \\
\hline A ggressivität & 10,1 & 7,3 & 7,3 & 8,4 & 3,2 & 4,8 & n.s. \\
\hline A ggressivität & 11,8 & 11,3 & 4,8 & 5,9 & 5,6 & 4,8 & n.s. \\
\hline $\begin{array}{l}\text { B eanspruchung } \\
\text { Körperliche }\end{array}$ & 5,9 & 8,9 & 3,2 & 13,6 & 8,9 & 5,6 & n.s. \\
\hline B eschwerden & 6,7 & 4,8 & 2,4 & 10,1 & 9,7 & 7,3 & n.s. \\
\hline G esundheitssorgen & 13,4 & 16,9 & 10,5 & 5,0 & 12,9 & 12,1 & n.s. \\
\hline G esundheitssorgen & 4,2 & 7,3 & 7,3 & 4,2 & 12,9 & 11,3 & n.s. \\
\hline G esundheitssorgen & 8,4 & 7,3 & 5,7 & 8,4 & 16,1 & 7,3 & n.s. \\
\hline G esundheitssorgen & 8,5 & 9,7 & 4,1 & 5,1 & 5,6 & 4,9 & n.s. \\
\hline G esundheitssorgen & 7,6 & 8,9 & 8,1 & 10,9 & 5,6 & 12,1 & n.s. \\
\hline G esundheitssorgen & 5,9 & 8,1 & 13,7 & 5,0 & 4,8 & 10,5 & $9,5^{*}$ \\
\hline Offenheit & 9,2 & 10,5 & 6,5 & 10,1 & 12,9 & 4,0 & n.s. \\
\hline Offenheit & 10,1 & 6,5 & 12 & 7,6 & 13,7 & 12,1 & n.s. \\
\hline O ffenheit & 9,2 & 10,5 & 8,9 & 8,4 & 9,7 & 10,5 & n.s. \\
\hline
\end{tabular}

Verhaltenstherapie 2000;10:166-176 


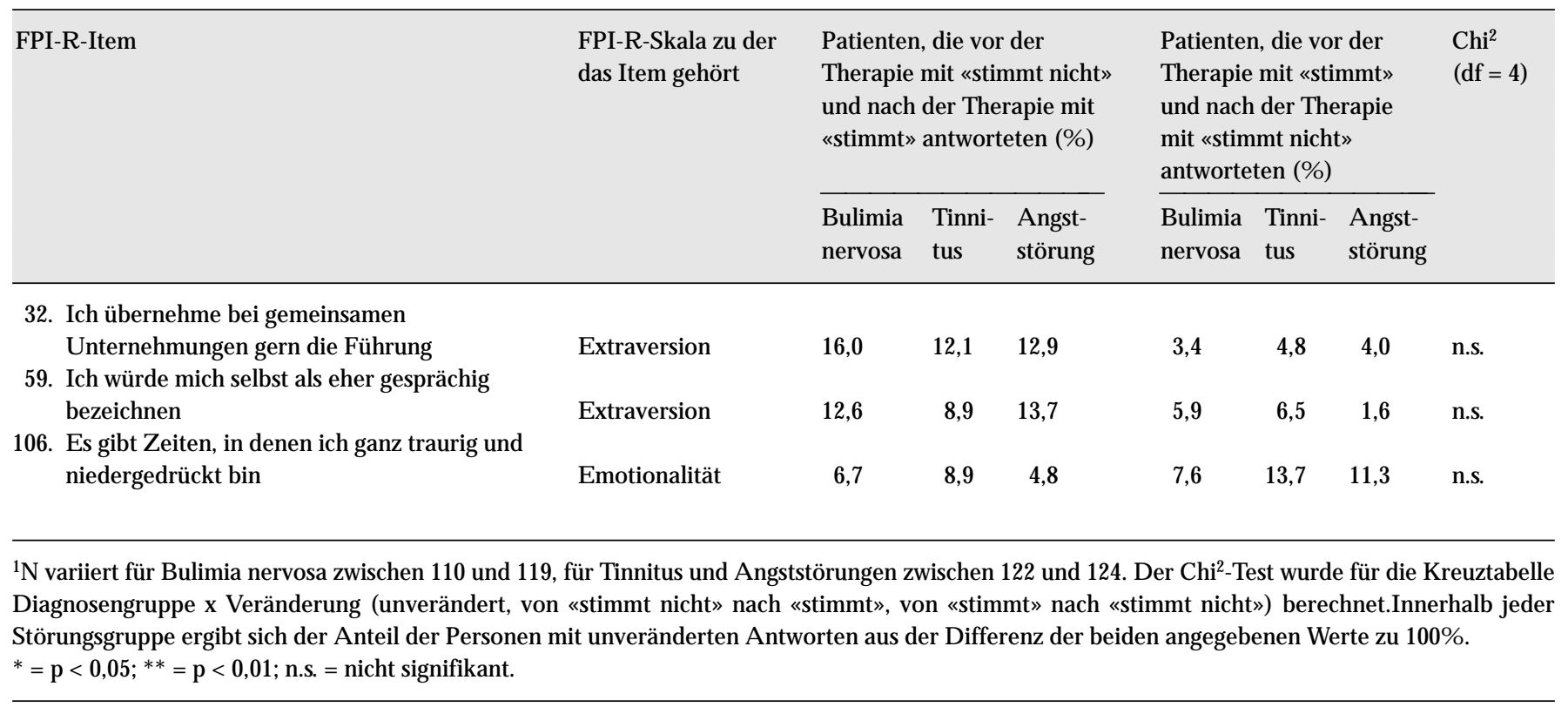

liegen H inweise vor, dass die Stabilität von Persönlichkeitsmerkmalen bei älteren Personen höher ist als bei jüngeren [z.B. Costa et al., 1980]. A uch bei einem Vergleich auf Itemebene zeigen sich die $U$ nterschiede zwischen unseren diagnostischen G ruppen. Die Stabilitätsindices waren in der Tinnitusgruppe und in der A ngststörungsgruppe höher als in der E ssstörungsgruppe. E in Vergleich der von uns ermittelten Stabilitätsindices auf I temebene mit anderen A rbeiten ist mangels dazu vorliegender Publikationen nicht möglich. E inzig die U ntersuchung von Schott und Lass [1971] hat die Stabilität der einzelnen FPI-Fragen an einer studentischen Stichprobe untersucht. $\mathrm{D}$ ie A utoren folgerten, dass die meisten Items relativ stabil sind, ohne entsprechende $Z$ ahlen vorzulegen.

Die Veränderungen im A ntwortverhalten bei Beginn und Ende der Therapie dürften die tatsächlichen Ä nderungen während der intensiven stationären Therapie ausdrücken. B eleg dafür ist, dass sich vor allem Items als instabil erwiesen, deren I nhalt einen B ezug zu den therapeutischen $M$ aßnahmen aufweist. B ei einer $R$ eihe von instabilen Items, die sich auf soziale Kompetenz und Leistung beziehen (z. B. Items 8, 13, 25 oder 48), verändern sich die A ntworten der Bulimia-nervosaGruppe im Sinne einer erhöhten Kompetenz und Leistungsbereitschaft. $D$ ies war nach einem stationär durchgeführten Selbstsicherheitstraining durchaus zu erwarten. Weitere Unterstützung erfährt diese Interpretation durch die E rgebnisse der A ngststörungsgruppe, welche im gleichen Sinne ihr A ntwortverhalten verändert. Für die Bulimia-nervosa-G ruppe gilt dies auch für die Veränderungen, die auf eine vermehrte emotionale Stabilität - sicherlich ein erklärtes Therapieziel - verweisen, wie z. B. die Items 19 oder 45. A Is dritter B ereich, den die instabilen Items erfassen, lässt sich eine erweiterte Freiheit von körperlichen B eschwerden und eine allgemein verbesserte $B$ efindlichkeit erkennen. $D$ ies ist der logische $E$ ffekt einer relativ langen, umfassenden stationären Therapie. A uch von der Itemformulierung her sind die instabilen Items eher auf eine Veränderung nach einer Therapie ausgerichtet. D ie vermehrte $B$ eantwortung von Items im Sinne einer positiven, erwünschten Richtung nach der Therapie könnte den U mstand reflektieren, dass die Patienten vor der E ntlassung sich eine positive Gestaltung ihres weiteren Leben zutrauen. D amit mögen I tems, die unbestimmte $\mathrm{H}$ äufigkeitsbegriffe oder auch häufiger verwendete Konjunktive - enthalten, hinsichtlich inres B edeutungsgehaltes vor und nach Therapie unterschiedlich bewertet werden, stehen doch keine absoluten Regeln, wie sie sich in den Wörtern nie oder immer ausdrücken, zur Entscheidung an. Möglicherweise erfolgt nach der Therapie ein differenzierteres A bwägen der A ntwort, sofern die Verwendung unbestimmter $\mathrm{H}$ äufigkeitswörter dies zulässt. D amit können A ntworten, deren Richtung von der Stimmung beim Ausfüllen des Fragebogens mit beeinflusst wird (z. B. Item 20 oder 25), gemäß dem hier aufgezeigten M uster ausfallen. D er Inhalt der stabilen Items unterstützt diese Interpretation. $\mathrm{H}$ ier findet sich ein seltener $\mathrm{G}$ ebrauch unbestimmter $\mathrm{H}$ äufigkeitswörter, außerdem sind öfters absolute Formulierungen ( vgl. Item 23 oder 32 ) verwendet. Interessanterweise gehören 3 der 6 stabilen Items, in denen unbestimmte H äufigkeitswörter verwendet werden, zur Skala Offenheit, welche von der A nlage her bereits Itemformulierungen enthält, die sehr allgemein gehalten sind. $D$ ies gilt auch für ein viertes I tem (16), welches ebenfalls sehr allgemein for- 
muliert ist. B etrachtet man die stabilen I tems, so gewinnt man den $\mathrm{E}$ indruck, dass die B eantwortung dieser I tems weniger von situationsgebundenen $E$ motionen und B efindlichkeitszuständen abhängig ist. D amit kann sich die spezielle emotionale B efindlichkeit nach einer stationären Therapie weniger auf die Beantwortung des FPI-R auswirken, woraus sich eine höhere Stabilität hinsichtlich des A ntwortverhaltens ergibt. I nhaltlich drückt sich dies in I temformulierungen mit wenigen unbestimmten $\mathrm{H}$ äufigkeitsbegriffen und der fehlenden Verwendung des Konjunktivs aus.

Die Zugehörigkeit der stabilen und instabilen Items zu den FPI-R -Skalen bestätigt die Schlüssigkeit unserer A rgumentation. Instabile Items gehören häufig zu den Skalen L eistungsorientierung und $\mathrm{G}$ ehemmtheit, also B ereichen, welche in der Therapie von Bulimia nervosa zu den zentralen Themen gehören. Stabile Items hingegen gehören häufiger zur Skala G esundheitssorgen, deren I tems eher allgemeine Lebensregeln ausformulieren.

B esonders soll darauf hingewiesen werden, dass die Veränderungen im Verlaufe des stationären A ufenthaltes stets in die $\mathrm{R}$ ichtung erfolgen, die man aufgrund der durchgeführten Therapie erwarten würde. D ies spricht dafür, dass echte Ä nderungen in den Persönlichkeitsmerkmalen erfolgt sind. G inge man davon aus, dass die erfassten Persönlichkeitsmerkmale stabil über den stationären A ufenthalt sind, die gemessenen U nterschiede damit lediglich A usdruck zufälliger Fluktuation (also Fehlervarianz im Sinne der M esstheorie), so müsste man Veränderungen sowohl im Sinne des erwarteten Therapieeffekts als auch in die entgegengesetzte $R$ ichtung finden. $D$ ies ist aber nicht der Fall.

$M$ ethodisch könnte eingewandt werden, dass wir ein relativ einfaches und hinsichtlich des Zufalls unkorrigiertes Stabilitätsmaß für die Items verwendeten. A Iternativ wäre die A nwendung etwa von Cohens K appa [Cohen, 1960] denkbar gewesen. $D$ ieses $M$ aß führt allerdings bei sehr schiefen A ntwortverteilungen zu deutlich niedrigeren Koeffizienten, verglichen mit unserem Verfahren. Dies hätte zu einer für unsere $Z$ wecke unangemessenen $O$ perationalisierung von Stabilität geführt. E inige Fragen wären als instabil charakterisiert worden, obwohl die Stabilität in unserem Sinne sehr offensichtlich ist. So hat das Item «l ch lebe mit mir selbst in Frieden und ohne innere Konflikte» einen Stabilitätskoeffizienten zwischen 79,0 und $92,4 \%$ und zählt damit zu den stabilen I tems. D er K appa-K oeffizient dagegen würde die Frage als instabiles Item erscheinen lassen.

$\mathrm{H}$ insichtlich der Veränderungen der M ittelwerte von B eginn zum Ende der Therapie könnte eingewendet werden, dass diese R esultate lediglich den Effekt einer Regression zur $M$ itte reflektieren. Um diese Möglichkeit zu untersuchen, haben wir zusätzlich $D$ aten einer Stichprobe von 41 adipösen Patienten der K linik R oseneck herangezogen. D iese Personen füllten einige Wochen vor A ufnahme in die Klinik R oseneck $\left(t_{0}\right)$, zu Beginn $\left(t_{1}\right)$ und zum Ende der stationären Therapie $\left(t_{2}\right)$ den FPI-R aus. Varianzanalytisch ergaben sich signifikante M esswiederholungseffekte (global über die 3 M esszeitpunkte) bei Lebenszufriedenheit, L eistungsorientierung, Gehemmtheit, Erregbarkeit, Offenheit, Extraversion und E motionalität. Betrachtet man jeweils zwei benachbarte $M$ esszeitpunkte (also $t_{0}$ und $t_{1}$, bzw. $t_{1}$ und $t_{2}$ ) müsste sich ein R egressionseffekt zur $M$ itte in einer $A$ nnäherung der zeitlich später erhobenen M ittelwerte zur M itte ausdrücken. Tatsächlich ist dies nicht der Fall, wenn man die jeweils zeitlich aufeinanderfolgenden $M$ esszeitpunkte ( $t_{0}$ versus $t_{1}$, bzw. $t_{1}$ versus $t_{2}$ ) vergleicht. Lediglich L ebenszufriedenheit verändert sich signifikant von der M essung vor A ufnahme in stationäre Therapie $\left(t_{0}\right)$ bis zu B eginn der Therapie $\left(t_{1}\right)$. A lle anderen Skalen zeigten keine signifikante Veränderung von $t_{0}$ bis $t_{1}$, so dass von einem stabilen A ntwortmuster ausgegangen werden kann.

A nders ist dies jedoch, nachdem die intensive Therapie durchgeführt worden ist. E s zeigen sich signifikante Veränderungen von B eginn $\left(t_{1}\right)$ bis $E$ nde $\left(t_{2}\right)$ der Therapie bei Lebenszufriedenheit, L eistungsorientierung, G ehemmtheit, O ffenheit, Extraversion und $E$ motionalität. D ieses M uster mit wenig Ä nderung vor Therapie und umfangreichen Ä nderungen während der Therapie bestätigt das Vorliegen eines Therapieeffekts und widerlegt das $A$ rgument der R egression zur M itte.

\section{Schlussfolgerung}

Die klinisch zu beobachtende Tatsache, dass sich Persönlichkeitsprofile, wie sie von gängigen Fragebogenverfahren erfasst werden, in kurzer Zeit verändern können, lässt sich durch spezifische Therapieeffekte erklären. E in Teil der Items des FPI$R$ erfasst einen Themenbereich, der in der stationären Therapie von Bulimia nervosa gezielt angegangen wird, so dass diese Veränderung eher zu erwarten ist. D amit ist allerdings nicht der Kern der Persönlichkeit verändert, sondern gerade nur der B ereich, der im R ahmen einer stationären Psychotherapie im Fokus der Verhaltensänderung steht und vom FPI-R erfasst wird. G rundsätzlich wird damit die Frage aufgeworfen, ob Persönlichkeitsinventare wie der FPI-R nur bedingt relativ zeitstabile Persönlichkeitszüge messen. $D$ ie hier berichteten E rgebnisse zeigen, dass sich sehr spezifische kurzfristige Ä nderungen im FPI-R im Rahmen gezielter Maßnahmen zur Verhaltensänderung nachweisen lassen.

M ethodisch lässt sich aus der vorliegenden Studie folgern, dass die Verwendung von Konjunktiven und unbestimmten $\mathrm{H}$ äufigkeitswörtern zu weniger konsistenten A ntworten über die Z eit führt, als absolut formulierte I tems. 


\section{Literatur}

A PA, A merican Psychiatric A ssociation: Diagnostic and Statistical $M$ anual for $M$ ental $D$ isorders. Washington D.C, D eutsche B earbeitung und $E$ inführung von K. Koehler und H. Saß: D iagnostisches und Statistisches M anual Psychischer Störungen D SM -III. Weinheim, B eltz, 1987.

Böhle A, von Wietersheim J, Wilke E Feiereis H: Die soziale I ntegration von Patientinnen mit A norexia nervosa und Bulimie. Z psychosom M ed 1991;37:282-291. B randstätter $\mathrm{H}$ : Stabilität und Veränderbarkeit von Persönlichkeitsmerkmalen. Z A rbeits- und Organisationspsychol 1989;33:12-20

Cohen J: A coefficient of agreement for nominal scales. E ducational and psychological M easurement 1960; 20:37-46.

Conley J]: The hierarchy of consistency: A review and model of longitudinal findings on adult individual differences in intelligence, personality and self opinion. Person Individ D ifferences 1984:5:11-26.

Costa PT, M cCrae R R , A renberg D : E nduring dispositions in adult males. J Person Soc Psychol 1980;38: 793-800.

D eter H-C, Schepank H : Patterns of self-definition of asthma patients and normal persons in the Freiburg Personality Inventory. Psychother Psychosom 1991;5: 47-56.
Fabra M, H esse C, B erzewski $\mathrm{H}$ : Kurzzeitkunsttherapie in der Psychiatrischen $\mathrm{K}$ risenintervention. M usikTanz- und Kunsttherapie 1991;2:97-108.

Fahrenberg J, H ampel R, Selg $\mathrm{H}$ : D as Freiburger Persönlichkeitsinventar, R evidierte Fassung FPI-R. G öt tingen, H ogrefe, 1984

Fahrenberg J, $\mathrm{H}$ ampel R, Selg $\mathrm{H}$ : D as Freiburger Persönlichkeitsinventar FPI. R evidierte Fassung FPI-R und teilweise geänderte Fassung FPI-A 1 (6. erg A ufl). Göttingen, H ogrefe, 1994.

Herrmann T: Lehrbuch der empirischen Persönlichkeitsforschung (4. A ufl). G öttingen, H ogrefe, 1984. H offmann M, Weithmann G, R othenbacher H: Persönlichkeit, soziale Integration und Therapieerfolg von A Ikoholikern. Sucht 1991;37:20-25

$\mathrm{K}$ inzl J, G ünther V, B iebl W, H interhuber $\mathrm{H}$ : A dipositas, Prädiktoren für Therapieeffizienz bzw. Therapieresistenz. A kt E rnähr 1989;14:22-26.

$\mathrm{K}$ ünsebeck $\mathrm{HW}$, Lempa $W$, Freyberger $\mathrm{H}$ : Kurz- und $L$ angzeiteffekte ergänzender Psychotherapie bei Patienten mit M orbus C rohn; in L amprecht F ( $\mathrm{H} \mathrm{rsg}$ ): Spezialisierung und Integration in Psychosomatik und Psy chotherapie. Deutsches Kollegium für psychosomatische M edizin, 6.-8. M ärz 1986. Heidelberg, Springer 1987, pp 253-262.
Langosch W, B rodner G : Persönlichkeits- und B efindlichkeitsveränderungen von $\mathrm{H}$ erzinfarktpatienten in A bhängigkeit von Erkrankungsdauer und Untersuchungssetting im Vergleich zu einer Kontrollgruppe chronisch K ranker. Z K lin Psychol 1984;13:204-220.

Paul $T$, Jacobi C: $E$ in ambulantes verhaltenstherapeutisches $\mathrm{G}$ ruppenprogramm bei Bulimia nervosa. Psychother med Psychol 1986;36:232-239.

Payk TR, Wachendorfer M : U ntersuchungen zur E ffizienz tagesklinischer Behandlung. Psychiat Prax 1987; 14:46-51.

Schott F, Lass U : U ntersuchungen zur Stabilität des FPI und zur Selbsteinschätzung auf den FPI-Skalen Diagnostica 1971;17:180-189.

Teusch L, Böhme $H$ : Was bewirkt ein stationäres Be handlungsprogramm mit gesprächspsychotherapeutischem Schwerpunkt bei Patienten mit A goraphobie und/oder Panik? Ergebnis einer 1-Jahres-K atamnese Psychother Psychosom med Psychol 1991;41:68-76. Tölle R, Peikert A , R ieke A : Persönlichkeitsstörungen bei M elancholiekranken. N ervenarzt 1987;58:227-236. 